\title{
Smart Design of Green Hydrogen Facilities: A Digital Twin-driven approach
}

\author{
Bruno Gerard ${ }^{1}$, Eduardo Carrera ${ }^{1,2}$, Olivier Bernard ${ }^{1,2}$, and Denis Lun ${ }^{1, *}$ \\ ${ }^{1}$ Envision Digital, 1 passerelle des reflets, 92400 Courbevoie, France \\ ${ }^{2}$ Capgemini Engineering, 2 Rue Paul Dautier, 78140 Vélizy-Villacoublay, France
}

\begin{abstract}
This work studies the potentials of Digital Twin solutions for the design of competitive and reliable green hydrogen facilities. A digital twin based on stochastic simulations is proposed to address the uncertainties associated with investment and operating costs, to increase confidence and stimulate investments. Several input assumptions are involved (i.e., capital and operational costs, energy consumption, available energy, among others) to analyse their influence on financial indicators. A set of facility designs with equipment redundancy, and thus different system availabilities, was proposed. Monte Carlo simulation method is chosen to propagate uncertainties onto the project bankability assessment. By applying the proposed methodology, the opportunity index and internal rate of return (IRR) are calculated. A sensibility analysis is also carried out. The simulations illustrate that the design of a facility can be optimized to achieve higher profits, based on a trade-off between investment and availability. This study concludes that digital twin solutions are an opportunity for reducing the uncertainties associated with green hydrogen facility design. Improvements to the proposed model can be achieved by performing a refined simulation, in relation to the calculation of system availability and maintenance costs.
\end{abstract}

\section{Introduction}

By 2050, global final energy consumption is expected to increase by about $30 \%$ and the production of electricity is projected to double [1]. However, currently, it is based on intensive exploitation of fossil energy sources. Approximately $81 \%$ of the world's energy consumption stem from coal, crude oil, and natural gas, which are at the origin of most of the greenhouse gas emissions that cause climate change [2]. Within the framework of the Paris Agreement aimed at a temperature increase of less than $2^{\circ} \mathrm{C}[3]$, numerous actions have been taken to achieve a decarbonized economy, mainly proposing an increase in the use of renewable energy sources [4]. However, this drastic change implies significant challenges to meet the growing demand for energy, from the point of view of security of supply, availability of resources and robustness of the power network.

Hydrogen has been identified as a key energy carrier to drive the energy transition and achieve the sustainable development goals $[5,6]$, being a long-term opportunity to move to cleaner energy and transport systems [7]. Through water electrolysis, green hydrogen is produced from renewable electricity. Among the main benefits are:
- Provision of long-term energy storage solutions for renewable energy sources [6].

- Diverse roles in different energy systems and industries (e.g., heat generation, low-carbon fuel supply, electricity generation, feedstock in chemical industry, etc.) [6].

- Hydrogen only emits water as a byproduct without any greenhouse gas emissions when it is used in a fuel cell [8].

- It possesses a high energy density (140 MJ/kg), being more than twice the energy density of typical energy carriers (e.g., $50 \mathrm{MJ} / \mathrm{kg}$ for methane) [8].

Nonetheless, green hydrogen technologies face multiple challenges. A major issue is the economic viability of water electrolysis using renewable electricity compared to other hydrogen production technologies (e.g., steam methane reforming) [9]. In this regard, significant cost reductions and efficiency improvements will be necessary before green hydrogen facilities can be economically competitive [10,11]. Furthermore, the Technology Readiness Level (TRL) of these technologies are at Level 3 to 7 [12], implying that a limited amount of historical data is available. This fact leads to poor estimates of system performance and thus represents investment risks. It is then crucial to evaluate the impact of input uncertainty on performance indicators related to decision-making.

\footnotetext{
* Corresponding author: denis.lun@envision-digital.com
} 
Digital Twin (DT) technologies have been successfully implemented for the intelligent use of available data. By using the internet of things (IoT), software simulation, and data analytics, DT technologies create a digital replica of a physical object or system [13]. Its use is gaining momentum due to rapidly evolving simulation and modelling capabilities, better coupling between interoperability and IoT sensors, and increased availability of infrastructure for the use of digital devices [14]. Furthermore, DT technologies have proven to offer advantages in accelerating technology growth, optimizing design, and improving return on capital [15-18]. Despite the potential offered by DT technologies, few attentions have been given to their application in green hydrogen facility design. To address these gaps, this works proposes a DT-driven approach based on stochastic simulations for the smart design of green hydrogen facilities.

This paper is divided into six sections. Following this introduction, a literature review is presented in Section 2. The main five steps of the methodology are described in Section 3. The case study chosen to illustrate the methodological framework is shown in Section 4. In Section 5, the main results and a sensibility assessment are analyzed. Finally, conclusions and perspectives are drawn in Section 6.

\section{Literature review}

Since its concept was introduced by Michael Grieves in 2002 [19], the Digital Twin technology has been applied to different fields of engineering [13-18]. They are the result of a continuous improvement of design, optimization and simulation methods used in industry, being part of what today is called Industry 4.0 paradigms [20]. DT concepts have been involved in energy systems and chemical industry mainly for the development of optimal management strategies [18,21,22], which is also the case for green hydrogen technologies:

- Ogumerem et al. propose an optimal thermal management for Proton Exchange Membrane Water Electrolysis (PEMWE) systems driven by DT technologies [23]. Their study shows that it can attenuate the long-term effects of high operating temperatures or rapid temperature changes on the polymer membranes.

- DT concepts for the optimal operation of hydrogen storage systems have been successfully developed [13]. The DT real-time connection to hydrogen vessels provides condition monitoring, allowing the operator to receive intelligent insights to reduce risk.

- Kang et al. presented a dynamic DT of a $25 \mathrm{~kW}$ Solid oxide fuel cells (SOFC) plant as a simulator to help operators safely and stably determine the operating conditions for a real commercial SOFC plant [24]. The results show that the on-site operation prediction is highly accurate, helping operators to determine operation strategies.

- Meraghni et al. propose a data-driven DT prognostics method for proton-exchange membrane fuel cells (PEMFCs). A DT is applied to establish an ensemble remaining useful life prediction system [25]. Its implementation achieves a high prediction accuracy. Furthermore, the predicted results are proved to be less affected even with limited measurement data.

Some studies focus on the design and simulation of hydrogen production plant components. For example, Wang et al. develops a high-accuracy data-driven surrogate model of proton exchange membrane fuel cells (PEMFCs) [26]. Their model has a comparable accuracyto a comprehensive 3D physical model. However, it considerably reduces the cost of computation and time.

As seen, methodologies based on data-driven DT approaches have been applied for the optimal management and simulation of green hydrogen technologies. However, this approach has not necessarily been applied to the design and evaluation of entire facilities. In the next section, a methodology to address some of these aspects is presented.

\section{Methodological framework}

A methodological framework for the smart design of green hydrogen facilities based on stochastic simulations and data-driven DT approach is presented. The methodology consists of five steps as described in Fig. 1.

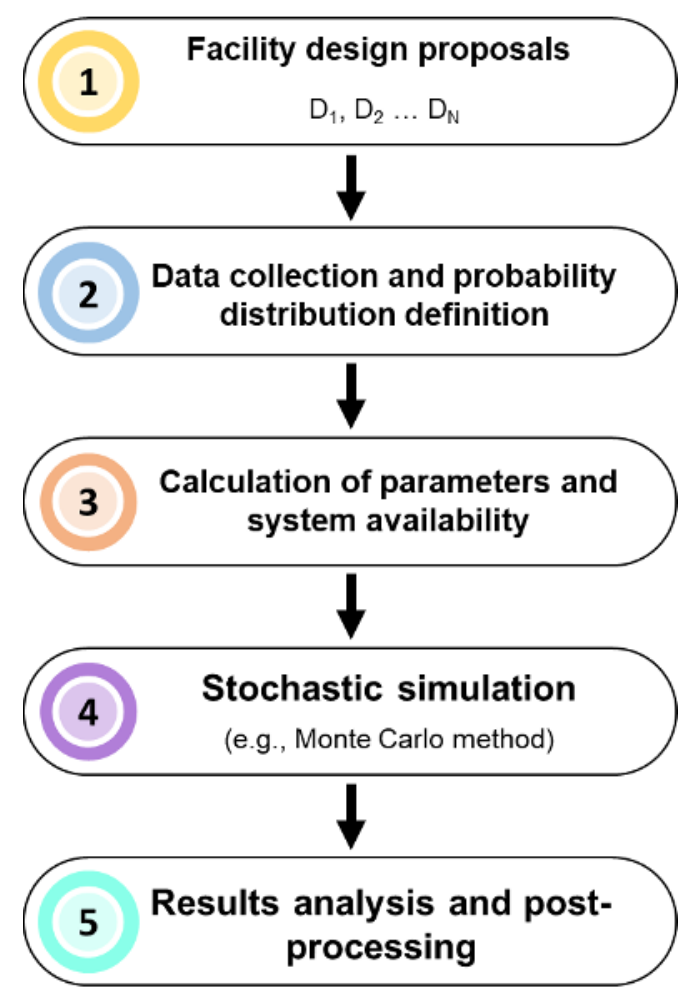

Fig. 1. Methodological framework 


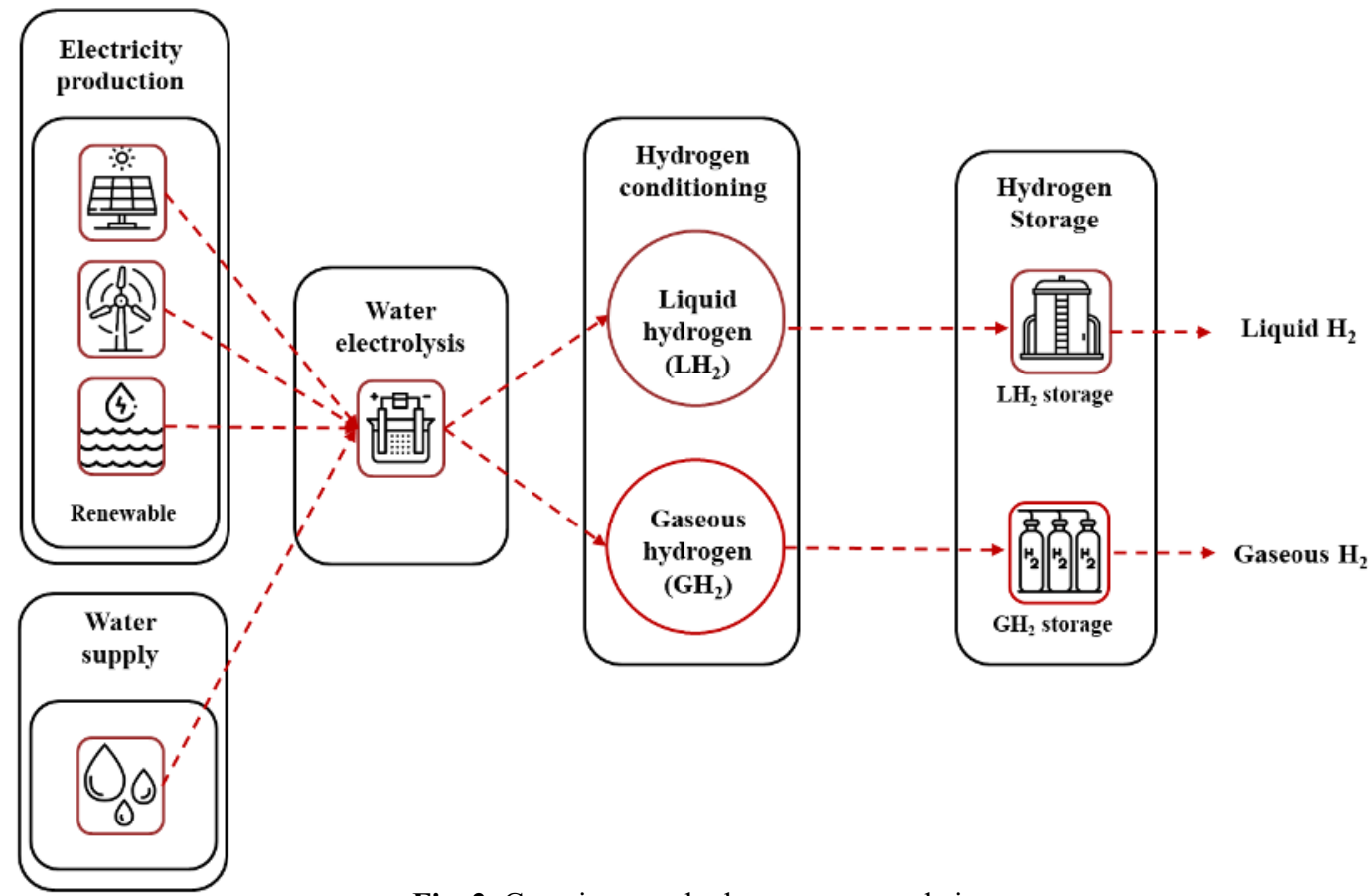

Fig. 2. Generic green hydrogen process chain

\subsection{Facility design proposals}

A green hydrogen production system typically consists of five main echelon [27], which are illustrated in Fig. 2. Renewable electricity and water are used to produce hydrogen through electrolysis. Hydrogen is then conditioned to obtain the desired physical form at specific conditions (i.e., pressure, temperature, residue gas composition, among others), which will determine the subsequent stages of the process chain. Hence, hydrogen could be compressed or liquefied, and stored.

Given the set of equipment that composes each of the echelons (e.g., compressors, pumps, heat exchangers, electrolyzers, etc.), several configurations are possible. Thus, different plant designs can be proposed with various level of redundancy of the equipment, which can be in series or in parallel. This leads to a trade-off between system availability and invested capital, being a key aspect to be addressed in order to increase the financial performance of the project. The following sections will address these issues.

\subsection{Data collection and probability distribution definition}

A set of techno-economic data is necessary to carry out the bankability analysis. It corresponds to the acquisition costs of each equipment, operating costs, main time to failure, main time to repair, process efficiencies, production rates, among others. It comes from direct monitoring of industrial systems. However, in case of insufficient information coming directly from operating facilities, data from manufacturers and expert consultation can be included. In this sense, some assumptions are made about the probabilistic distribution function (PDF) of the data involved (i.e., triangular, Gaussian, constant, among others) [28].
The subsequent incorporation of data from industrial projects operating under real conditions allows to refine previous assumptions, which leads to updating the PDF of inputs. For this purpose, there is a set of strategies such as Bayesian network modelling, which have demonstrated their applicability to this type of self-learning process $[29,30]$.

\subsection{Calculation of system availability}

The calculation of the system availability of the design facilities is the next step in the proposed methodology. The availability of a repairable system can be defined as the probability that the system will be operational at a given time "t" [31]. An analytical approach to calculating availability is presented in Eq. (1) to (5). The Main Time to Failure $\left(\mathrm{MTTF}_{\mathrm{i}}\right)$ and Main Time to Repair $\left(\mathrm{MTTR}_{\mathrm{i}}\right)$ for each equipment " $i$ " are used in Eq. (1) and (2) to calculate their failure $\left(\lambda_{\mathrm{i}}\right)$ and repair rate $\left(\mu_{\mathrm{i}}\right)$, respectively. In this case, they are assumed to be constant. These parameters are used to determine the availability of each equipment through Eq. (3). Finally, the system availability is calculated considering series $\left(A_{s}\right)$ or parallel $\left(A_{p}\right)$ configurations by using Eq. (4) and (5), respectively.

$$
\begin{gathered}
\lambda_{i}=\frac{1}{M T T F_{i}} \\
\mu_{i}=\frac{1}{M T T R_{i}} \\
a_{i}(t)=\frac{\lambda_{i}}{\lambda_{i}+\mu_{i}}+\frac{\mu_{i}}{\lambda_{i}+\mu_{i}} \exp \left(-\lambda_{i}+\mu_{i}\right) t
\end{gathered}
$$




$$
\begin{gathered}
A_{s}(t)=\prod_{\substack{i=1 \\
n}}^{n} a_{i}(t) \\
A_{p}(t)=1-\prod_{i=1}^{n}\left(1-a_{i}(t)\right)
\end{gathered}
$$

\subsection{Stochastic simulation}

Stochastic simulations are used to consider uncertainty on several input parameters. Thus, the simulation output will have an inherent variability due to the stochastic nature of the system under study, which is referred to as stochastic uncertainty [32]. Renewable energy systems exhibit these characteristics due to the intermittency of the renewable energy sources (e.g., solar and wind sources). The algorithms used to deal with this type of problem are well known and are generally based on statistical approaches [32].

This study proposes the use of Monte Carlo simulations as stochastic method [33], which is well-known and successfully applied to similar energy systems [34,35]. It can be summarized as follow:

- Define input parameters and probability distribution functions (as described in Section 3.2. and 3.3.).

- Find the cumulative distribution function (CDF) for each parameter.

- Sample from the CDF using a random number generator (between 0 and 1 ).

- Perform simulations with the generated samples to obtain the CDF of the outputs. The number of simulations will depend on the level of precision required.

\subsection{Results analysis and post-processing}

The application of a stochastic simulation, in this case, the Monte Carlo method, allows to obtain the CDF of the outputs. Several outputs can be evaluated for further analysis. In this case, the internal rate of return (IRR) and the "opportunity index" are proposed as the main performance indicators.

\subsubsection{Internal rate of return}

The internal rate of return (IRR) is the discount rate that would yield a net present value (NPV) of zero over the planning period. A zero NPV value means that the discounted cashflows generated by the project are equivalent to the cashflows obtained with an investment on a bank account serving an interest rate equivalent to IRR [36].

\subsubsection{Opportunity index}

The opportunity index corresponds to the probability of achieving a value equal to or lower than the desired output value.

\section{Case study}

In order to demonstrate the usefulness of the proposed methodology, an analysis was carried on a project located in the south of Spain. This region is one of the most suitable for the deployment of photovoltaic systems in Europe, thanks to its highest levels of solar radiation. As results, it is one of the regions that have been allocated the highest volumes of the EU funds to promote solar energy projects [37].

The project to be evaluated consists of the production of green hydrogen from solar electricity (i.e., photovoltaic). The data considered involve each of the echelon in the green hydrogen production chain: energy production, water supply, water electrolysis, hydrogen conditioning and buffer storage. The data comes from consultation with manufacturers, experts and industrial data. The main parameter inputs are given below:

- The technology used for the electrolysis of water corresponds to pressurized alkaline electrolysis.

- The facility's capacity is $100 \mathrm{MWh}$, which corresponds to 20 electrolyzers of $5 \mathrm{MW}$ each.

- $100 \%$ of the electricity used comes from solar energy source.

- The final physical form of hydrogen is gaseous.

- Hydrogen is compressed from 30 to 70 bar.

- The target IRR is 5\%.

- The lifespan of the project is 35 years.

Three green hydrogen facility design configurations are tested, considering redundancy in key equipment (i.e., electrolyzers and compressors), which implies a trade-off between CAPEX and system availability. They are shown in Table 1.

Table 1. Green hydrogen facility design configurations

\begin{tabular}{cl}
\hline \multicolumn{1}{c}{ Design } & \multicolumn{1}{c}{ Description } \\
\hline --- 1 (Base case) & $\begin{array}{l}\text { Project in the south of Spain } \\
\text { Design 1 with 20\% increase in } \\
\text { hydrogen production capacity. }\end{array}$ \\
--- 2 (Hypothetical A) & $\begin{array}{l}\text { Design 2 with doubled } \\
\text { compression capacity }\end{array}$ \\
\hline
\end{tabular}

\section{Results}

The results obtained by applying the proposed methodology are shown in this section. Table 2 presents the CAPEX and system availability of each design. System availability in Design 2 and 3 increases by 2.5 and $3.5 \%$ compared to the baseline design, respectively. Nevertheless, an increase in availability implies a higher investment. CAPEX in Design 2 and 3 rises by $20 € / \mathrm{kW}$ (5.4\%) and $70 € / \mathrm{kW}(19 \%)$ compared to Design 1, respectively. 
Table 2. Availability and CAPEX for each design (hydrogen production)

\begin{tabular}{ccc}
\hline Design scenario & $\begin{array}{c}\text { System availability } \\
(\%)\end{array}$ & $\begin{array}{c}\text { CAPEX } \\
(€ / \mathrm{kW})\end{array}$ \\
\hline---1 (Base case) & 96.0 & 370 \\
---2 (Hypothetical A) & 98.5 & 390 \\
---3 (Hypothetical B) & 99.5 & 440 \\
\hline
\end{tabular}

As indicated in Section 3.5, the IRR and the opportunity index were defined as the main performance indicators. Fig. 2 (a) then shows the opportunity index vs IRR for the base design. It reflects a potential business for the project under study. It is observed that An IRR of 5\% leads to an opportunity rate of $70 \%$, thus showing the robustness of the basic design. To study the most critical drivers to achieve the targeted IRR, a sensitivity analysis was carried out, which is presented in Fig. 3 (b). It shows that the investment costs of electrolyzers, their power consumption, and the annual solar energy available represent the three critical parameters affecting the IRR, which can have an impact of up to $+/-0.23 \%$. This implies that in order to obtain competitive green hydrogen plants,

a)

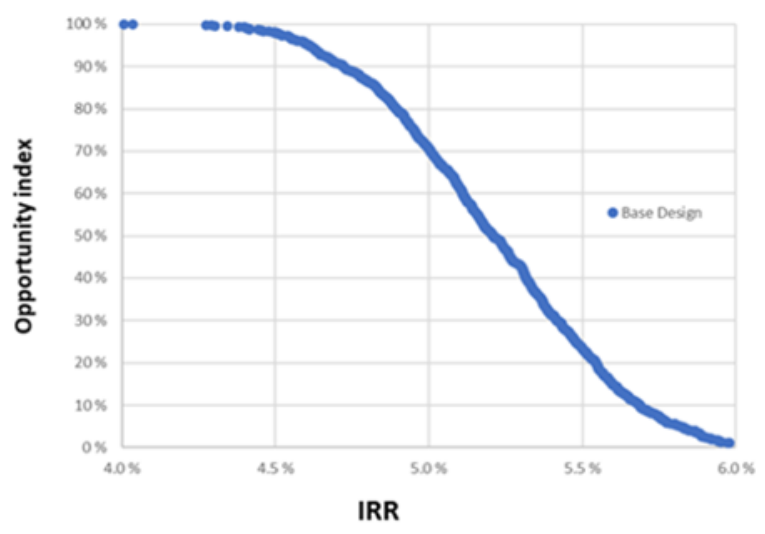

b)

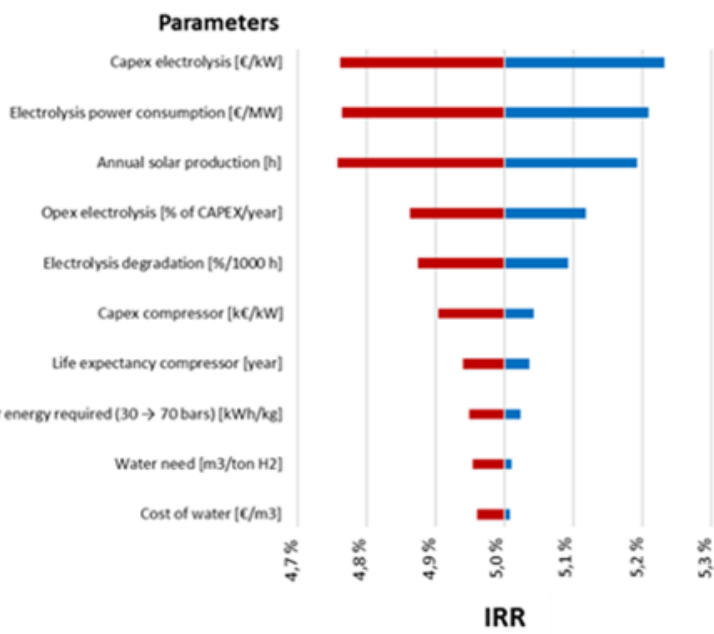

Fig. 3. (a) Opportunity index as a function of IRR for the base case (Design 1); (b) Sensitivity analysis it is necessary to prioritize the reduction of the investment costs of the electrolyzers, which has also been indicated by other studies [38]. The energy consumed in the electrolysis process has also been identified in the literature as a key factor in increasing the profitability of green hydrogen production systems $[11,38,39]$. This should be a core aspect in the technological development of the future generation of electrolyzers. In addition, this parameter, as well as the annual solar energy available, also implies an operational optimization of the system. The development of technologies such as DT can be useful for such purposes, which has already been highlighted in other studies [23-25].

A comparison between the three proposed designs is carried out in Fig. 4. Their IRR and opportunity index are shown in Fig. 4 (a). "Design 2" allows to obtain a higher opportunity rate despite having a higher CAPEX (see Table 2), thanks to an increase in the system availability compared to the "Design 1". "Design 3" decreases the opportunity index and therefore the probability of achieving higher IRR values. A comparison of the three configurations was made at P90 (chance ratio equal to $90 \%$ ). The IRR as a function of CAPEX is shown in Fig. 4 (b). The IRR in "Design 2" increases by $0.24 \%$, while it decreases by $0.43 \%$ in "Design 3".

a)

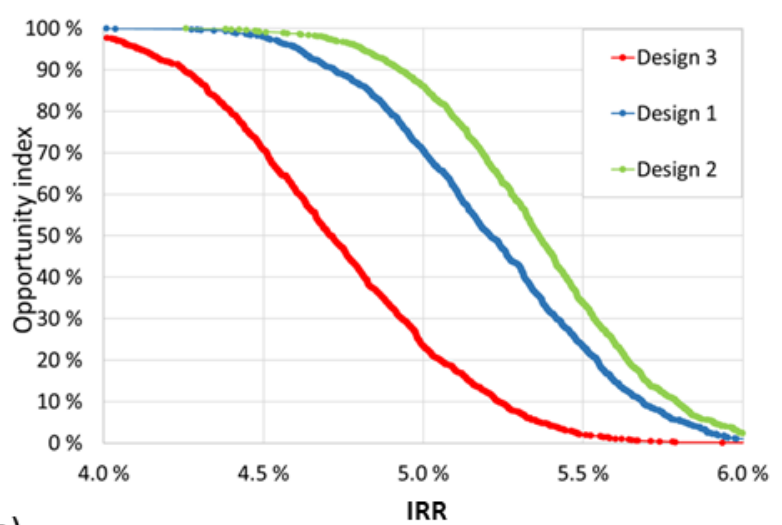

b)

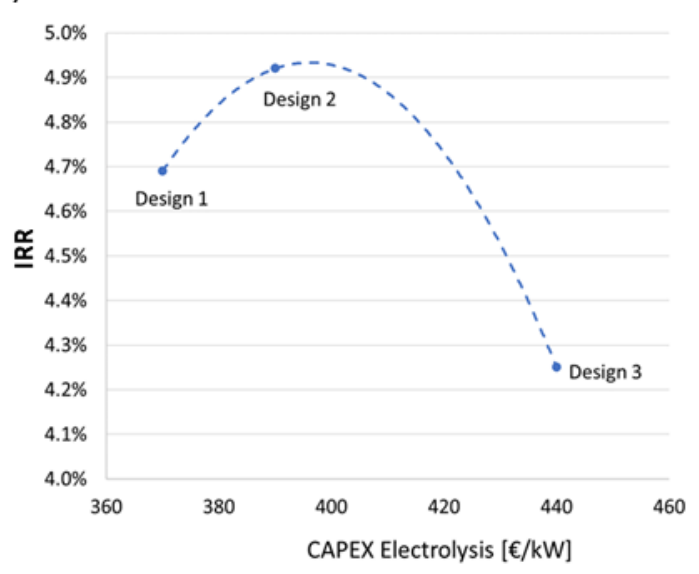

Fig. 4. (a) Opportunity index as a function of IRR for the three designs proposed; (b) IRR as a function of CAPEX. 
According to this analysis, "Design 2" would be the green hydrogen facility design to be considered. This illustrates that an increase in the availability of the electrolyzers is enough to improve the financial performance of the hydrogen facility. In this sense, one aspect to consider would be the possibility of evaluating different hydrogen production technologies (i.e., PEMWE and solid oxide electrolysis cells), in order to compare the results with the precedent designs.

\section{Conclusions}

With the outputs of the proposed methodological framework applied to the green hydrogen facility design, we were able to demonstrate that an approach driven by DT technologies and stochastic analysis generates substantial value-added insights to decision-makers. Firstly, the approach developed allows addressing the uncertainty inherent in the input parameters and its consequences on key financial performance indicators. Secondly, the probability of reaching a given value using an indicator such as the opportunity index provides confidence intervals. Thus, the risk associated with the project can be mitigated. Finally, different designs can be proposed and evaluated, which allows to find trade-offs between the capital invested and the system availability. Consequently, the proposed approach contributes to optimal, coherent, and robust decision making.

The usefulness of the methodology was shown in a case study. Three green hydrogen facility design were tested. When comparing the designs, it was determined that "Design 2" presented the best business opportunity, thanks to an improved trade-off between capital investment and system availability compared to the base case. The calculation of its opportunity index shows that higher IRR can be attained with lower risk. Besides, a sensitivity analysis was performed to identify the parameters that have the major impact on the IRR. The capital cost of electrolyzers is the main parameter that needs to be improved to increase business opportunities and is a key issue for the development of the next generations of electrolyzers.

Future research will be conducted on the treatment of the input data when it will come from direct monitoring of different hydrogen production systems, since the simulation output data, and hence the performance measures, are directly affected by the probability distributions (i.e., input models) from which the sample paths are generated in simulation [32]. In this sense, a key aspect to be addressed will be the appropriate selection of probability models that will adequately characterize the stochastic behavior of the system, in order to reduce uncertainty. Among the major issues to be considered are the calculation of system availability and operating costs.
The authors would like to thank the team of experts from Corporate Value Associates (CVA), Capgemini Engineering and Envision Digital, whose comments contributed to enrich the work carried out.

\section{References}

1. IAEA, Energy, Electricity and Nuclear Power Estimates for the Period up to 2050 (Vienna, 2020)

2. IEA, Key World Energy Statistics 2020 (Paris, 2020)

3. United Nations, Paris Agreement (Paris, 2015)

4. IRENA, (2020)

5. European Commission, (2020)

6. A. Lewandowska-Bernat and U. Desideri, Appl. Energy 228, 57 (2018)

7. T. da Silva Veras, T. S. Mozer, D. da Costa Rubim Messeder dos Santos, and A. da Silva César, Int. J. Hydrogen Energy 42, 2018 (2017)

8. S. Shiva Kumar and V. Himabindu, Mater. Sci. Energy Technol. 2, 442 (2019)

9. E. Carrera and C. Azzaro-Pantel, Comput. Chem. Eng. 153, 107386 (2021)

10. T. Egeland-Eriksen, A. Hajizadeh, and S. Sartori, Int. J. Hydrogen Energy 46, 31963 (2021)

11. E. Carrera and C. Azzaro-Pantel, Comput. Aided Chem. Eng. 50, 1859 (2021)

12. R. Pinsky, P. Sabharwall, J. Hartvigsen, and J. O’Brien, Prog. Nucl. Energy 123, 103317 (2020)

13. A. Jaribion, S. H. Khajavi, M. Öhman, A. Knapen, and J. Holmström, in (2020), pp. 369-375

14. J. Leng, D. Wang, W. Shen, X. Li, Q. Liu, and X. Chen, J. Manuf. Syst. 60, 119 (2021)

15. C. K. Lo, C. H. Chen, and R. Y. Zhong, Adv. Eng. Informatics 48, 101297 (2021)

16. Y. Wang, X. Wang, and A. Liu, Procedia CIRP 93, 198 (2020)

17. M. Liu, S. Fang, H. Dong, and C. Xu, J. Manuf. Syst. 58, 346 (2021)

18. E. Örs, R. Schmidt, M. Mighani, and M. Shalaby, in 2020 IEEE Int. Conf. Eng. Technol. Innov. (2020), pp. 1-8

19. M. Grieves, White Pap. 1, 1 (2014)

20. H. Lasi, P. Fettke, H.-G. Kemper, T. Feld, and M. Hoffmann, Bus. Inf. Syst. Eng. 6, 239 (2014)

21. A. Ebrahimi, in 2019 IEEE 28th Int. Symp. Ind. Electron. (2019), pp. 1059-1066

22. E. O'Dwyer, I. Pan, R. Charlesworth, S. Butler, and N. Shah, Sustain. Cities Soc. 62, 102412 (2020)

23. G. S. Ogumerem and E. N. Pistikopoulos, J. Process Control 91, 37 (2020)

24. J.-L. Kang, C.-C. Wang, D. S.-H. Wong, S.-S. Jang, and C.-H. Wang, J. Taiwan Inst. Chem. Eng. 118, $60(2021)$ 
25. S. Meraghni, L. S. Terrissa, M. Yue, J. Ma, S. Jemei, and N. Zerhouni, Int. J. Hydrogen Energy 46, 2555 (2021)

26. B. Wang, G. Zhang, H. Wang, J. Xuan, and K. Jiao, Energy AI 1, 100004 (2020)

27. E. Carrera and C. Azzaro-Pantel, Chem. Eng. Sci. 246, 116861 (2021)

28. J. S. Arora, Introduction to Optimum Design, Fourth edi (Elsevier, Iowa, 2017)

29. M. Borunda, O. A. Jaramillo, A. Reyes, and P. H. Ibargüengoytia, Renew. Sustain. Energy Rev. 62, 32 (2016)

30. T. Adedipe, M. Shafiee, and E. Zio, Reliab. Eng. Syst. Saf. 202, 107053 (2020)

31. Y. Hou, M. Sallak, and W. Schön, IFACPapersOnLine 28, 1315 (2015)

32. C. G. Corlu, A. Akcay, and W. Xie, Oper. Res. Perspect. 7, 100162 (2020)

33. A. F. Magnússon, R. Al, and G. Sin, Comput.
Aided Chem. Eng. 48, 451 (2020)

34. E. J. da S. Pereira, J. T. Pinho, M. A. B. Galhardo, and W. N. Macêdo, Renew. Energy 69, 347 (2014)

35. Y. Gu, X. Zhang, J. Are Myhren, M. Han, X. Chen, and Y. Yuan, Energy Convers. Manag. 165, 8 (2018)

36. E. H. Macdonald, Handb. Gold Explor. Eval. 553 (2007)

37. C. Perpiña Castillo, F. Batista e Silva, and C. Lavalle, Energy Policy 88, 86 (2016)

38. H. Böhm, A. Zauner, D. C. Rosenfeld, and R. Tichler, Appl. Energy 264, 114780 (2020)

39. A. Buttler and H. Spliethoff, Renew. Sustain. Energy Rev. 82, 2440 (2018) 